\section{Cold storage of platelets in additive solution: the impact of residual plasma in apheresis platelet concentrates}

\author{
Irene Marini, ${ }^{1 *}$ Konstanze Aurich, ${ }^{2 *}$ Rabie Jouni, ${ }^{1}$ Stefanie Nowak-Harnau, ${ }^{1}$ \\ Oliver Hartwich, ${ }^{2}$ Andreas Greinacher, ${ }^{2}$ Thomas Thiele ${ }^{2,7}$ and Tamam \\ Bakchoul ${ }^{1,2, \mp}$ \\ ${ }^{1}$ Centre for Clinical Transfusion Medicine, Medical Faculty of Tübingen, University of \\ Tübingen and ${ }^{2}$ Institute of Immunology and Transfusion Medicine, University of \\ Greifswald, Germany
}

*Both authors shared first authorship. FBoth laboratories contributed equally to this work

\section{ABSTRACT}

P latelet transfusion has become essential therapy in modern medicine. Although the clinical advantage of platelet transfusion has been well established, adverse reactions upon transfusion, especially transmission of bacterial infection, still represent a major challenge. While bacterial contamination is favored by the storage of platelets at room temperature, cold storage may represent a solution for this important clinical issue. In this study, we aimed to clarify whether plasma has protective or detrimental effects on cold-stored platelets. We investigated the impact of different residual plasma contents in apheresis-derived platelet concentrates, stored at $4^{\circ} \mathrm{C}$ or room temperature, on platelet function and survival. We found that platelets stored at $4^{\circ} \mathrm{C}$ have higher expression of apoptosis marker compared to platelets stored at room temperature, leading to accelerated clearance from the circulation in a humanized animal model. While cold-induced apoptosis was independent of the residual plasma concentration, cold storage was associated with better adhesive properties and higher response to activators. Interestingly, delta $(\delta)$ granule-related functions, such as ADP-mediated aggregation and CD63 release, were better preserved at $4^{\circ} \mathrm{C}$, especially in $100 \%$ plasma. An extended study to assess cold-stored platelet concentrates produced under standard care Good Manufacturing Practice conditions showed that platelet function, metabolism and integrity were better compared to those stored at room temperature. Taken together, our results show that residual plasma concentration does not have a cardinal impact on the cold storage lesions of apheresis-derived platelet concentrates and indicate that the current generation of additive solutions represent suitable substitutes for plasma to store platelets at $4^{\circ} \mathrm{C}$.

\section{Introduction}

Transfusion of platelet concentrates (PCs) is essential to reduce blood loss after traumatic injury or to maintain a safe platelet (PLT) count during chemotherapy. PCs are currently stored at room temperature $\left(20-24^{\circ} \mathrm{C}\right)$ with constant agitation to ensure adequate PLT recovery, survival, and sufficient therapeutic efficacy. However, storage at room temperature (RT) not only compromises functionality both in vivo and in vitro ('PLT storage lesions'), but also increases the potential risk of microbial growth in case of contamination..$^{1-6}$ For these reasons, the shelf life of PLTs is limited to 4-7 days, depending on country specific guidelines. ${ }^{2}$ However, despite limited storage time, the incidence of bacterial contamination of PCs remains high, ranging from 1 to 10 per 50,000 units, which is a major drawback of PLTs stored at RT for clinical use.

Cold storage of PCs at $4^{\circ} \mathrm{C}$ could be an option to reduce the risk of bacterial growth. ${ }^{78}$ Recent studies reported that cold-stored PLTs are functionally and metabolically superior to those stored at RT.1-11 A potential limitation of cold storage is the poorer recovery and survival of PLTs after transfusion. However, this remains controversial. Some studies have shown a decrease in survival of cold-stored PLTs
Ferrata Storti Foundation
Haematologica 2018
Volume 103(2):207-214

\section{Correspondence:}

tamam.bakchoul@med.uni-tuebingen.de

Received: April 16, 2018.

Accepted: August 9, 2018.

Pre-published: August 16, 2018.

doi:10.3324/haematol.2018.195057

Check the online version for the most updated information on this article, online supplements, and information on authorship \& disclosures: www.haematologica.org/content/104/1/207

(C)2019 Ferrata Storti Foundation

Material published in Haematologica is covered by copyright. All rights are reserved to the Ferrata Storti Foundation. Use of published material is allowed under the following terms and conditions:

https://creativecommons.org/licenses/by-nc/4.0/legalcode. Copies of published material are allowed for personal or internal use. Sharing published material for non-commercial purposes is subject to the following conditions: https://creativecommons.org/licenses/by-nc/4.0/legalcode, sect. 3. Reproducing and sharing published material for commercial purposes is not allowed without permission in writing from the publisher. 
in comparison to RT-stored PLTs, ${ }^{12-14}$ while other investigators reported that PLTs stored at $4^{\circ} \mathrm{C}$ can survive in the circulation for several days. ${ }^{15,16}$

In this context, the residual plasma content of PLT storage media may be relevant. Early studies have investigated cold storage of PLTs in plasma and have reported poor recovery and survival. ${ }^{17}$ Based on these studies, the concept of cold storage had been abandoned in routine clinical practice. Recently, with the availability of PLT storage in additive solutions (PAS), the cold storage of PCs has seen something of a renaissance. Storage in PAS was suggested to maintain better PLT quality and provide protection from storage lesions with the possibility of prolonging PC shelf life. ${ }^{18-20}$ However, it is still unclear whether reduced plasma content improves cold storage of PCs. Furthermore, it is not known whether recovery and survival of PLTs are better after cold storage in PAS compared to storage at RT.

In this study, we investigated the impact of different residual plasma concentrations in apheresis-derived platelet concentrates (APCs) stored at $4^{\circ} \mathrm{C}$ or at RT. We aimed to clarify whether plasma has protective or detrimental effects on cold-stored PLTs. Moreover, we assessed in vitro PLT quality and function in APCs to define the optimal balance between cold storage in plasma and additive solution. We then initiated a validation study of coldstored APCs produced under Good Manufacturing Practice (GMP) conditions with $35 \%$ residual plasma to verify the feasibility of PLT cold storage for clinical use.

\section{Methods}

\section{Preparation of apheresis platelet concentrates}

Apheresis-derived platelet concentrates were collected from healthy volunteers according to the German guidelines for hemotherapy. Ten individuals donated two units of APCs collected with FENWAL AMICUS (Amicus, Fresenius Kabi, Bad Homburg, Germany) and stored in plasma or in PAS (SSP+, Macopharma, Langen, Germany) at different final plasma concentrations $[100 \%$ (Plasma-APC), $35 \%$ (PAS-35-APC) or $20 \%$ (PAS-20-APC)] at $4^{\circ} \mathrm{C}$ and RT. See the Online Supplementary Methods for further details. Finally, PAS-35-APCs were produced under GMP-conditions (12 healthy male donors) as described, ${ }^{21}$ and stored at $\mathrm{RT}$ and $4^{\circ} \mathrm{C}$.

\section{In vivo studies}

To assess the survival of PLTs derived from APCs, we used the NOD/SCID mouse model as described previously. ${ }^{22,23}$ See the Online Supplementary Methods for further details.

\section{Measurement of glycan changes}

Glycan pattern was analyzed by flow cytometer (FC) (Navious, Beckman Coulter) using ricinus communis agglutinin (RCA, 0.5 $\mu \mathrm{g} / \mathrm{mL}$, Vector, Burlingame, CA, USA) which binds beta ( $\beta$ )-galactose, as described in the Online Supplementary Methods.

\section{Apoptosis}

Platelets from APCs were washed, resuspended with $1 \mathrm{mM}$ $\mathrm{CaCl}_{2}$, and stained with Annexin V-FITC (Beckman Coulter) for 60 minutes ( $\mathrm{min}$ ) at RT. Freshly isolated PLTs were incubated with $10 \mu \mathrm{M}$ ionomycin (Abcam, Cambridge, UK) and used as positive control. See the Online Supplementary Methods for further details.

\section{Platelet adhesion}

Coverslips (Corning, New York, USA) were coated with 100 $\mu \mathrm{g} / \mathrm{mL}$ of fibrinogen (Sigma Aldrich, Munich, Germany) or colla- gen (Horm Collagen-Takeda, Linz, Austria). PLTs from APCs $\left(1 \times 10^{8} \mathrm{PLTs} / \mathrm{mL}\right)$ were seeded on coverslips and incubated for 1 hour (h) at RT with TRAP (0.1 mM, Hart Biologicals, Hartlepool, UK). The adherent cells were fixed with $4 \%$ paraformaldehyde (PFA) for $20 \mathrm{~min}$ at RT. Images were captured from 5 different microscopic fields/coverslips (x100, Olympus IX73, Tokyo, Japan).

\section{Platelet activation and granule release}

Platelets $\left(10 \times 10^{6} / \mathrm{mL}\right)$ were incubated with TRAP $(40 \mu \mathrm{M})$ for 30 $\min$ at $37^{\circ} \mathrm{C}$, and fixed with $4 \%$ PFA for 20 min at RT. The expression of CD62P (CLB-Thromb/6, Beckman Coulter) and CD63 (CLB-Gran/12, Beckman Coulter) was determined by flow cytometry (FC) as well as the conformational changes of glycoproteins (GPs) IIb/IIIa complex by PAC-1-antibody (BD Bioscience).

\section{Platelet aggregation}

Platelet function was analyzed by FC and light transmission PLT aggregation assay (LTA) using a 4-channel-aggregometer (Labitec, Ahrensburg, Germany). See the Online Supplementary Methods for further details.

\section{Hypotonic shock reaction}

Hypotonic shock reaction (HSR) was determined by LTA. Percentage of HSR was calculated as described in the Online Supplementary Appendix.

\section{Statistical analysis}

Statistical analyses were performed using GraphPad Prism 7 (La Jolla, USA). A $t$-test was used to analyze normally distributed results. Non-parametric tests were used when data failed to follow a normal distribution as assessed by the D'Agostino and Pearson omnibus normality test. Group comparison was performed using the Wilcoxon rank-sum test and the Fisher exact test with categorical variables. In the case of a small number of experiments $(<10)$, the group comparison was performed using the $t$-test. $P<0.05$ was considered statistically significant.

\section{Ethics}

All studies involving human subjects were approved by the ethics committees of the University Hospital of Tübingen and the Universitätsmedizin Greifswald. Animal studies were approved by the state animal ethics committees of Baden-Württemberg and Mecklenburg-Vorpommern.

\section{Results}

\section{Platelet survival after cold storage at different plasma concentrations}

At storage day 7, PLTs were injected into the NOD/SCID mice and the survival of PLTs stored at different conditions was compared in pairs. To enable statistical analysis between PLTs from different donors, the relative survival of Plasma-APC stored at RT was considered as 1.0. Fewer PLTs were found in the circulation when APCs were stored at $4^{\circ} \mathrm{C}$ compared to RT, regardless of whether PLTs where stored in 35\% residual plasma [PAS-35-APC stored at $4^{\circ} \mathrm{C} v s$. RT mean \pm Standard Error of mean (SEM): $1.05 \pm 0.02$ vs. $0.63 \pm 0.16$, respectively, $P=0.04$; Figure $1 \mathrm{~A}]$ or in $20 \%$ residual plasma (PAS-20-APC $0.58 \pm 0.05$ vs. $0.34 \pm 0.05$, respectively, $P=0.01$; Figure $1 \mathrm{~B}$ ).

Regarding the plasma content, similar survival curves were observed at each temperature when PLTs were stored in 35\% plasma compared to $100 \%$ plasma (Online 
Supplementary Figure S1A). In contrast, PLTs stored in PAS with only $20 \%$ residual plasma were cleared faster from the mouse circulation compared to those stored in 100\% plasma, and this was even more pronounced in coldstored PLTs (Online Supplementary Figure S1B).
Cold-induced apoptosis rather than desialylation is associated with accelerated clearance of cold-stored platelets, irrespective of residual plasma

Cold storage of PLTs in plasma has been reported to induce desialylation of PLTs. Therefore, we tested the
A

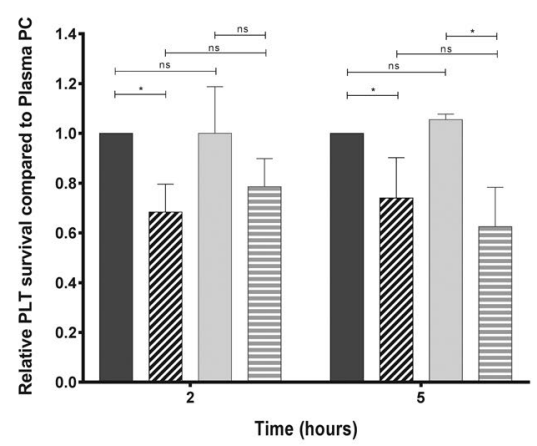

B

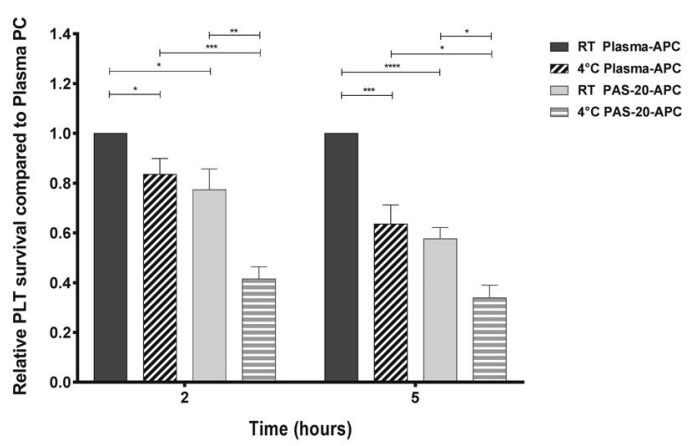

Figure 1. Relative platelet survival after cold storage at different residual plasma concentrations (PC). Apheresis platelet concentrates (APCs) were collected, split and stored at room temperature (RT) or at $4^{\circ} \mathrm{C}$, either in $100 \%$ plasma (Plasma-APC, black bars) or in platelet additive solution (PAS, gray bars) at two different residual plasma concentrations: (A) 35\% (PAS-35-APC) and (B) 20\% (PAS-20-APC). Platelets (PLTs) were obtained from APCs at storage day 7 and administered into the mouse circulation via the lateral tail vein. Survival of human PLTs in mouse was analyzed by collecting murine blood after two and five hours. For statistical comparison between different groups, the percentage of circulating human PLTs was normalized to Plasma-APC in each corresponding experiment (Plasma-APC was considered 1.0). Data are shown as mean \pm Standard Error of Mean. ${ }^{*} P<0.05 ; * \star P<0.01 ; * * * P<0.001 ; * * * * P<0.0001$. ns: not significant ( $\mathrm{n}=5$ ). Survival curves are available in Online Supplementary Figure S1.

A

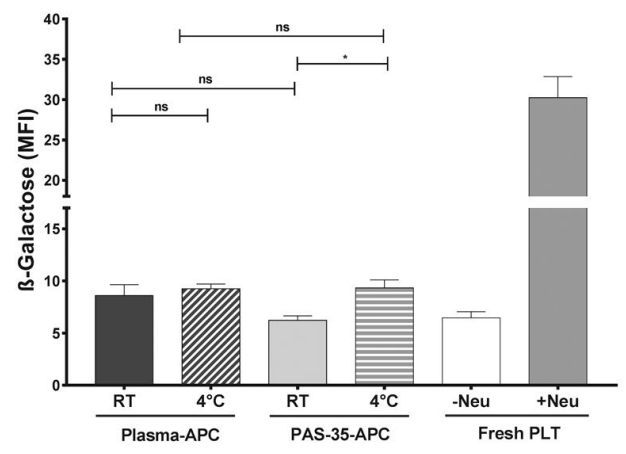

C

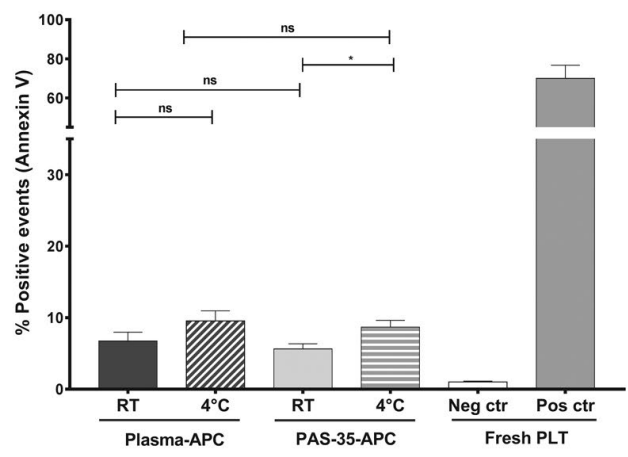

B

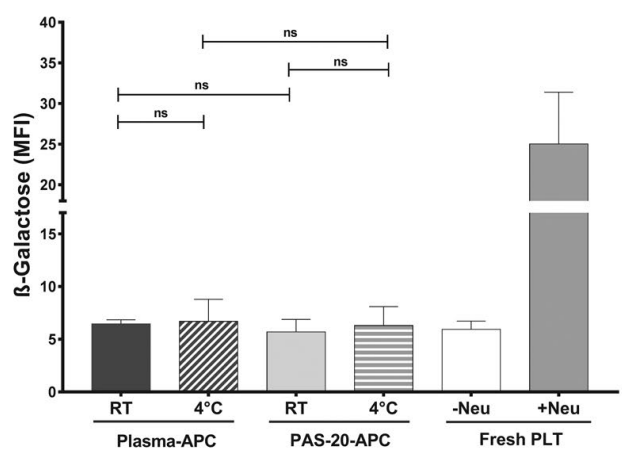

D

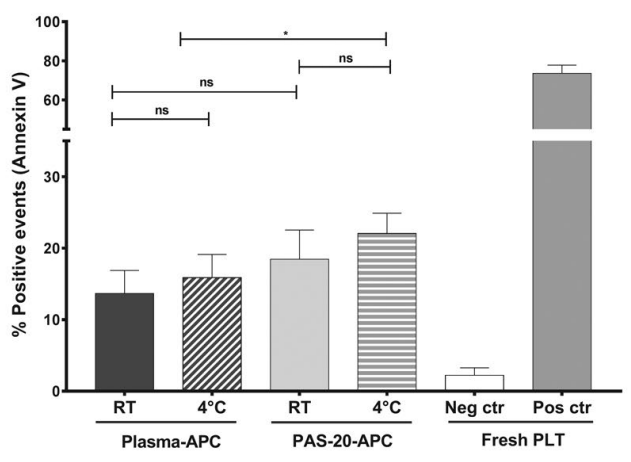

Figure 2. Desialylation and apoptosis after cold storage. Platelet (PLT) desialylation (A and B) and apoptosis (C and D) were analyzed after seven days of storage, at room temperature (RT) or $4^{\circ} \mathrm{C}$, in apheresis platelet concentrates (APCs) containing (A and C) $35 \%$ plasma (PAS-35-APC), (B and D) 20\% plasma (PAS-20-APC) or $100 \%$ plasma (Plasma-APC). Desialylation was determined by measurement of FITC-labeled RCA $(0.5 \mu \mathrm{g} / \mathrm{mL})$ that binds to beta ( $\beta$ )-galactose using flow cytometetry. Fresh PLTs incubated with or without neuraminidase (Neu) were used as positive and negative control, respectively. The percentage of apoptotic cells was measured using FITC-labeled Annexin V. As positive (Pos ctr) and negative control (Neg ctr), freshly isolated PLTs incubated in the presence or in the absence of lonomycin were used. Data are shown as mean \pm Standard Error of Mean of fluorescence intensity (MFI) and percentage of positive events, respectively. $* P<0.05$. ns: not significant $(n=4)$. 
exposure of desialylated GPs in PLTs stored at different plasma concentrations. In the lectin binding assay (LBA), neither storage temperature nor plasma content had a major effect on $\beta$-galactose expression (Figure $2 \mathrm{~A}$ and $\mathrm{B}$ ). Only PLTs from PAS-35-APCs stored for seven days at $4^{\circ} \mathrm{C}$ showed a slightly higher exposure of $\beta$-galactose on their surface compared to those stored at RT.

In contrast, higher exposure of phosphatidylserine was always detected on the surface of cold-stored PLTs (percentage of Annexin $V$ positive events mean \pm SEM: PAS-35APC $9 \pm 1$ vs. $7 \pm 1$, respectively, $P=0.033$; Figure 2C; PAS20 -APC $22 \pm 4$ vs. $17 \pm 5$, respectively, $P=0.317$; Figure $2 \mathrm{D}$ ). The cold-induced increase in the exposure of phosphatidylserine was not correlated to the residual plasma concentration indicating that cold storage of APCs affects PLT survival by inducing cell apoptosis in a plasma-inde-
A

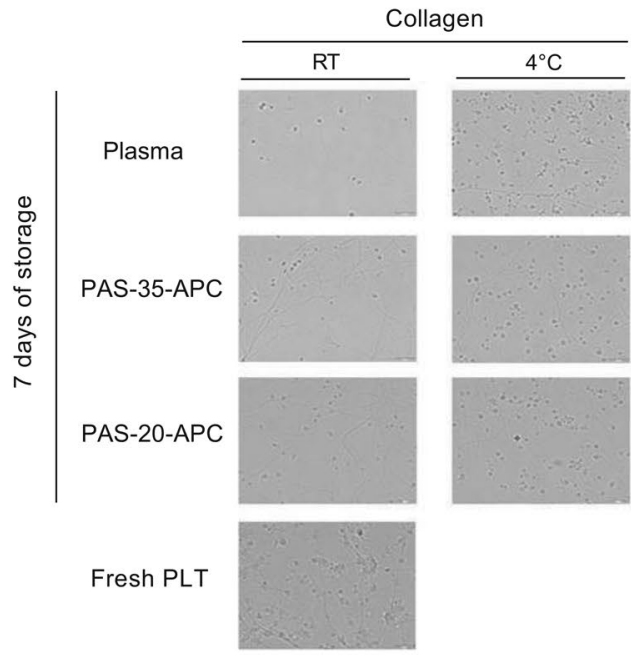

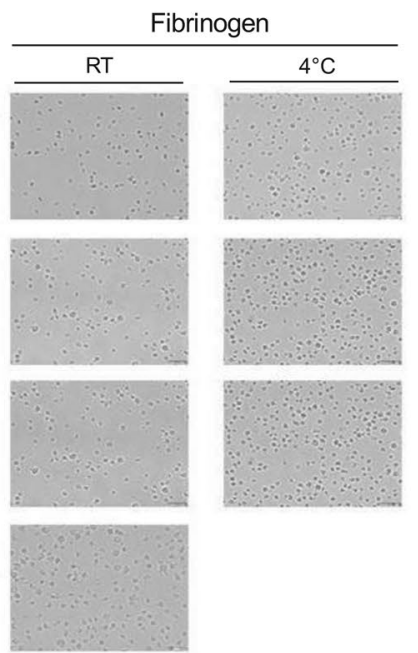

B

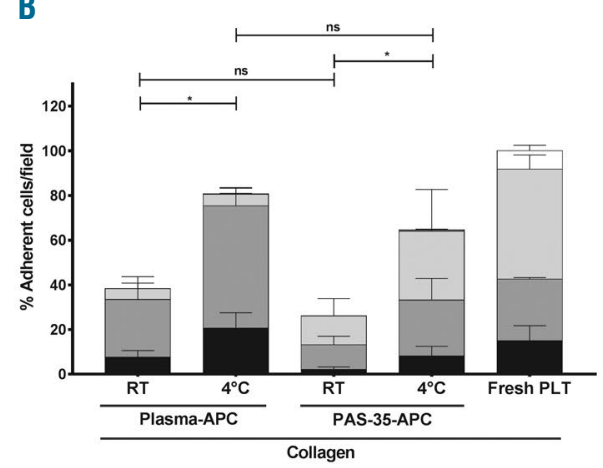

D

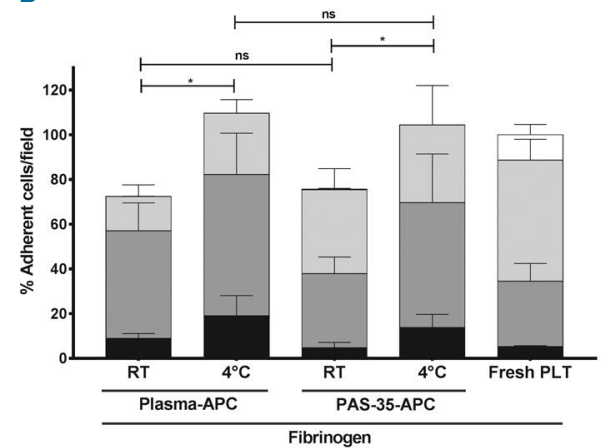

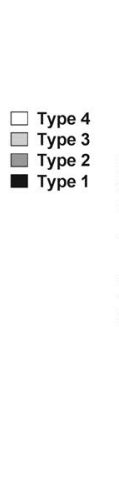

C
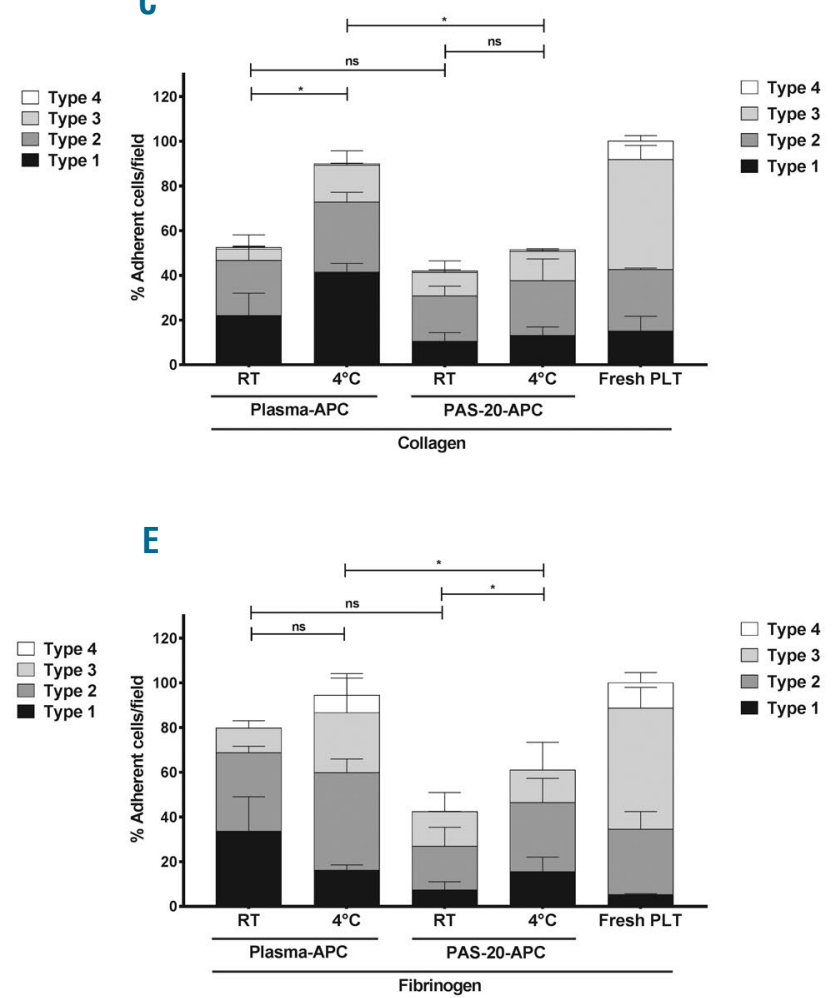

$\mathrm{E}$

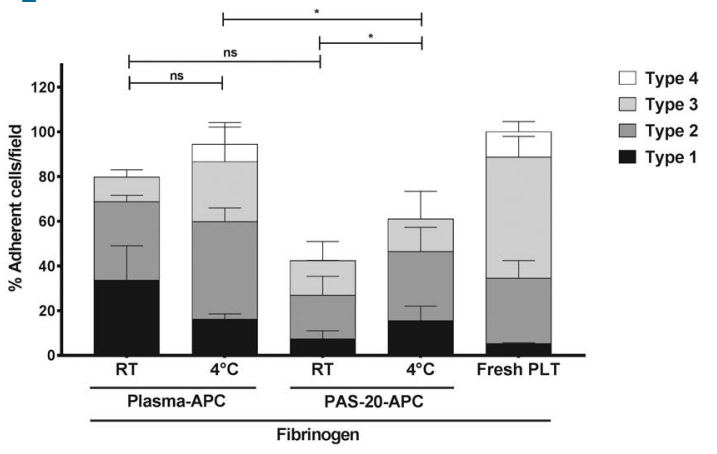

Figure 3. Platelet adhesion to collagen and fibrinogen after storage at room temperature (RT) or $4{ }^{\circ} \mathrm{C}$. (A) Representative images of adherent platelets (PLTs) from different apheresis platelet concentrates (APCs) after seven days of storage (scale bar $10 \mu \mathrm{m}$ ). PLTs from APCs containing $100 \%$ plasma (Plasma-APC), 35\% plasma (PAS-35-APC) and 20\% plasma (PAS-20-APC) were allowed to adhere to collagen (B and C) or fibrinogen (D and E) surfaces in the presence of TRAP. The number and types of adherent PLTs were determined from 5 different microscopic fields per coverslip (see Online Supplementary Figure S2 and Online Supplementary Table S1A and $B$ for further details). Freshly isolated PLTs were used as positive control (100\%). Data are shown as mean \pm Standard Error of Mean. ${ }^{*}<<0.05$. ns: not significant $(n=3)$. 
pendent manner. While similar Annexin V binding was observed on PLTs from PAS-35-APCs and Plasma-APCs stored at both temperatures, cells stored in $20 \%$ residual plasma (PAS-20-APC) showed higher exposure of the apoptosis marker phosphatidylserine, particularly when PLTs were cold-stored (Figure 2D).

\section{Effect of residual plasma on adhesion of cold-stored platelets}

The adhesion of TRAP-activated PLTs was analyzed after seven days of storage. More PLTs from APCs stored at $4^{\circ} \mathrm{C}$ adhered to collagen and fibrinogen compared to PLTs stored at RT (Figure 3A). This effect was statistically significant for PLTs stored in $100 \%$ and $35 \%$ plasma (Figure $3 \mathrm{~B}$ and D). Moreover, the individual percentage of each spreading pattern (type 1, 2, 3 and 4) (Online Supplementary Figure S2) was always higher after cold storage, regardless of plasma volume, but without reaching statistical significance (Online Supplementary Table S1A and $B)$. Cells stored in the presence of $35 \%$ plasma showed similar adhesive response to both proteins compared to $100 \%$ plasma (Figure 3B and D). In contrast, significantly fewer PLTs adhered to both proteins from cold-stored PAS-20-APCs compared to plasma-APCs (percentage of adherent PLTs to collagen, mean \pm SEM: $13 \pm 4$ vs. $41 \pm 4$, $P=0.033$; Figure $3 C$ ) and to fibrinogen (mean \pm SEM: $16 \pm 7$ vs. $16 \pm 2$, respectively, $P=0.028$; Figure $3 \mathrm{E}$ ). This suggests that an excessive reduction in plasma volume to lower than 35\% impairs PLTs adhesive functions, especially when PLTs were stored at $4^{\circ} \mathrm{C}$.

\section{Plasma diminishes loss of $\delta$ granule secretion during cold storage}

Secretion of granule content in response to PLT activation is required for an efficient hemostatic function. All stored PLTs had a reduced release of granule markers compared to freshly isolated ones. However, storage at $4^{\circ} \mathrm{C}$ improved CD63 release, especially when PLTs were stored in plasma: fold increase of CD63 after activation with TRAP mean \pm SEM: plasma-APC versus PAS-35-APC, $3.88 \pm 0.65$ versus $2.71 \pm 0.27$, respectively, $P=0.145$ (Figure $4 \mathrm{~A})$; and plasma-APC versus PAS-20-APC, $4.75 \pm 1.18$ versus $2.75 \pm 0.75$, respectively, $P=0.045$ (Figure $4 B$ ). Storage solution and temperature had only a minor impact on the storage lesions causing diminished CD62P expression (Figure $4 \mathrm{C}$ and D).

\section{Validation of cold PLT functions in stored APCs}

As a step towards optimization of cold storage in routine clinical practice, we next evaluated the effect of cold storage using PAS-35-APCs produced under GMP conditions to demonstrate the quality of cold-stored PLTs prepared for clinical use throughout extended storage for ten days.

Platelet aggregation in response to collagen was significantly higher when PLTs were stored at $4^{\circ} \mathrm{C}$ (Figure 5A). A
A

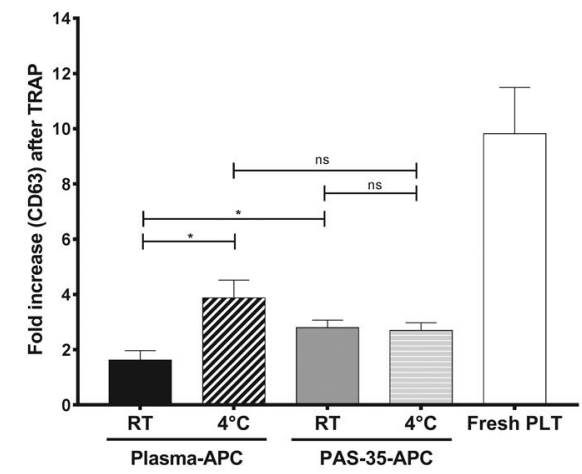

C

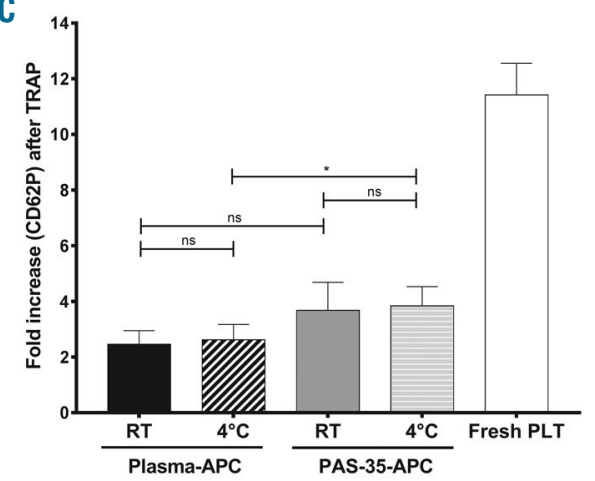

B

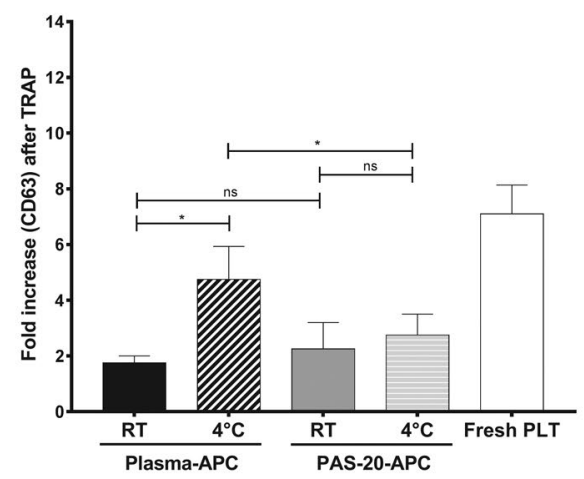

D

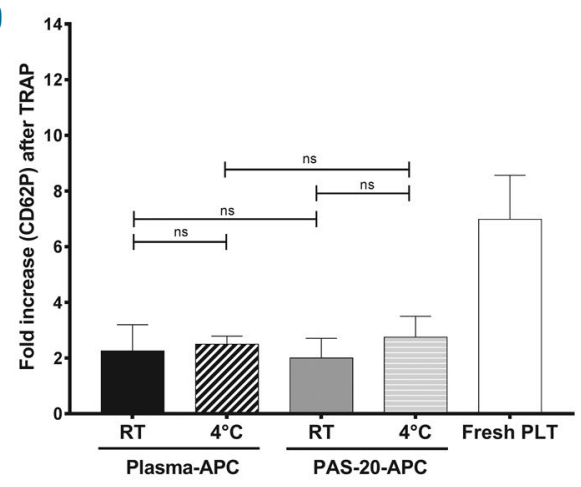

Figure 4. The impact of storage temperature and residual plasma concentration on granules release upon activation. After seven days of storage, $C D 63$ ( $A$ and $B$ ) and CD62P (C and D) expressions were determined for apheresis platelet concentrates (APCs) containing 100\% plasma (Plasma-APC), 35\% plasma (PAS-35-APC) and $20 \%$ plasma (PAS-20-APC) after activation with TRAP. Freshly isolated platelets (PLTs) were used as control for both markers. Data are shown as mean \pm Standard Error of Mean of fold increase of mean florescence intensity compared to buffer as baseline. ${ }^{*} P<0.05$. ns: not significant ( $\left.n=4\right)$. RT: room temperature. 
significantly higher response to collagen was found after four days of storage at $4^{\circ} \mathrm{C}$ in comparison to RT (percentage of maximal aggregation after collagen, mean \pm SEM $67 \pm 6$ vs. $22 \pm 5$, respectively, $P<0.0001$ ), consistent with their better adhesion to collagen (Figure 3 ). In addition, the $\delta$-granule-dependent response to ADP was reduced less during storage in PAS at $4^{\circ} \mathrm{C}$ compared to RT (Figure 5B). Ristocetin-mediated PLT agglutination was slightly lower at $4^{\circ} \mathrm{C}$ compared to RT (Figure $5 \mathrm{C}$ ). Along the same lines as for PLT aggregation, after activation with TRAP a slightly higher expression of CD62P and CD63 was observed on cold-stored PLTs and the gradual reduction in PLT response was slower (Figure 6A and B). In contrast, no significant impact on the ability of conformational change in the integrin alpha $(\alpha) \mathrm{IIb} / \beta(\beta)$ III was found during storage, regardless of temperature (Figure 6C). Furthermore, similar glucose consumption, and lactate release was found in both storage conditions (Online Supplementary Figure $S 3 A$ and $B$, respectively). Finally, although hypotonic shock reaction decreased in a time-dependent manner, it was always higher when PLTs were stored at RT compared to $4^{\circ} \mathrm{C}$ (Figure 6D).

\section{Discussion}

In the present study, we investigated the impact of residual plasma concentration in APCs on cold storage lesions, including in vivo survival and in vitro hemostatic functions. Despite the reduced in vivo survival of coldstored PLTs, we showed that cold storage not only preserves PLT response to aggregation agonists, but also maintains their adhesion to thrombogenic surfaces better than RT storage. Substituting $65 \%$ of plasma with PAS did not have any important impact on cold-induced storage lesions. However, poor survival and functional results were observed when plasma concentration was further reduced to $20 \%$.

Attempts to store APCs at $4^{\circ} \mathrm{C}$ were impeded by a shorter survival of cold-stored PLTs. In our study, PLTs from APCs stored at $4^{\circ} \mathrm{C}$ always showed inferior survival curves compared to those stored at RT. This confirmed previous reports on accelerated clearance of cold-stored PLTs. ${ }^{13,14}$ Data from an animal model suggested that desialylation of GPIb is responsible for the accelerated elimination of coldstored PLTs. ${ }^{13,24}$ However, we found similar $\beta$-galactose exposure on RT- and cold-stored PLTs. This fits the results of clinical studies which showed that reconstitution of sialylation in human PLTs does not prevent the accelerated clearance of cold-stored APCs. ${ }^{25}$ Interestingly, we found that cold storage triggers phosphatidylserine exposure indicating higher PLT apoptosis. Although the differences were not always significant, there is an obvious trend. This may indicate that impaired in vivo survival of cold-stored PLTs is actually caused by apoptosis-related mechanisms. In fact, cold storage had previously been found to induce GPIb $\alpha$-clustering which makes the receptors an initiation site for PLT apoptosis and macrophage recognition. ${ }^{26}$ The inhibition of prostacyclin has been suggested to reduce cold-induced changes in $\mathrm{GPIb}{ }^{27}$ which could be emphasized by our results. Interestingly, coldinduced apoptosis in our study was not accompanied by higher spontaneous expression of $\mathrm{P}$-selectin or CD63, indicating that apoptosis and activation during PLT storage are mediated by two independent mechanisms. Therefore, selective targeting of cold-induced apoptosis may provide a new approach to reduce PLT storage lesions. In fact, it was recently reported that cold storage of PLTs enhances
A

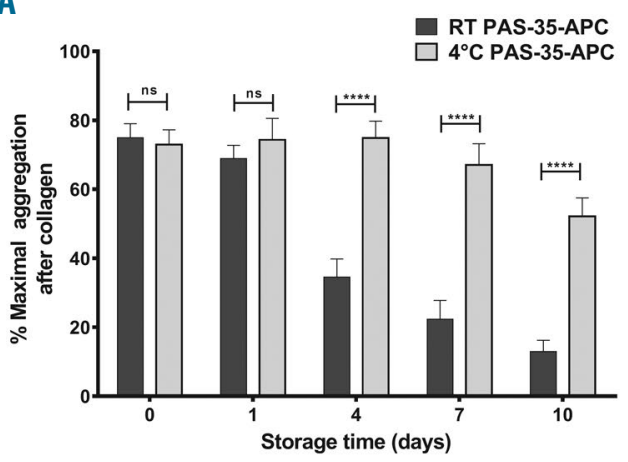

C

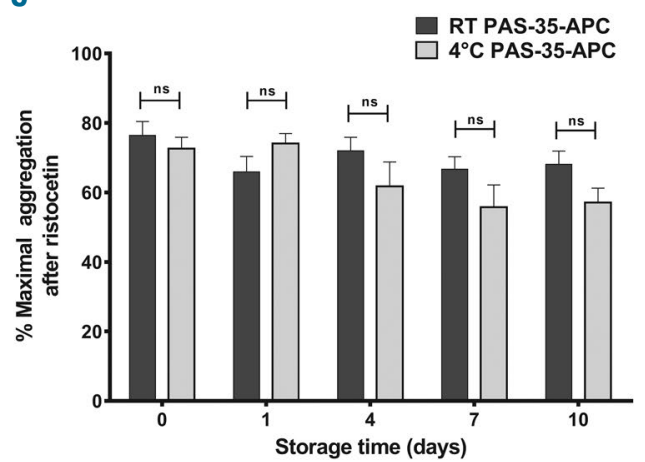

B

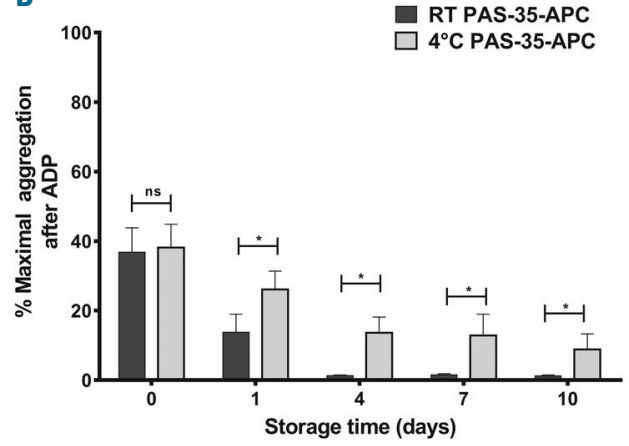

Figure 5. Functional analyses of cold-stored apheresis platelet concentrates. Apheresis platelet concentrates (APCs) were produced under Good Manufacturing Practice. conditions and stored in platelet additive solution (PAS) at a residual plasma concentration of 35\% (PAS-35-APC) at room temperature (RT) or $4^{\circ} \mathrm{C}$. The maximal aggregation ability of platelets (PLTs) was determined after activation using three inductors: (A) collagen $(8 \mu \mathrm{g} / \mathrm{mL}),(B)$ ADP $(80 \mu \mathrm{M})$ and $(C)$ ristocetin $(1.5 \mu \mathrm{M})$ (agglutination). Data are shown as mean \pm Standard Error of Mean. ${ }^{*} P<0.05 ; * * * * P<0.0001$. ns: not significant $(n=4)$. 
A

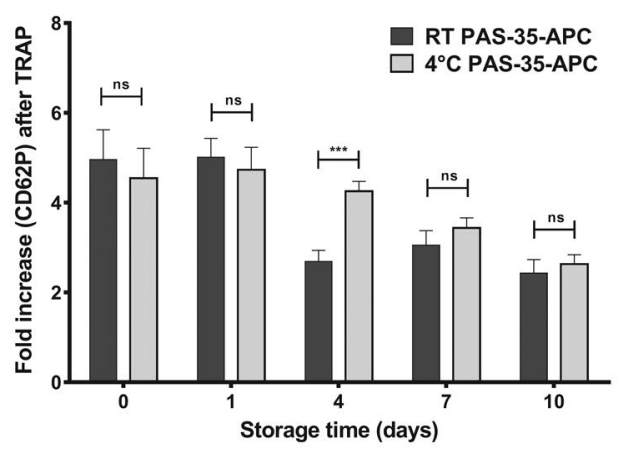

C

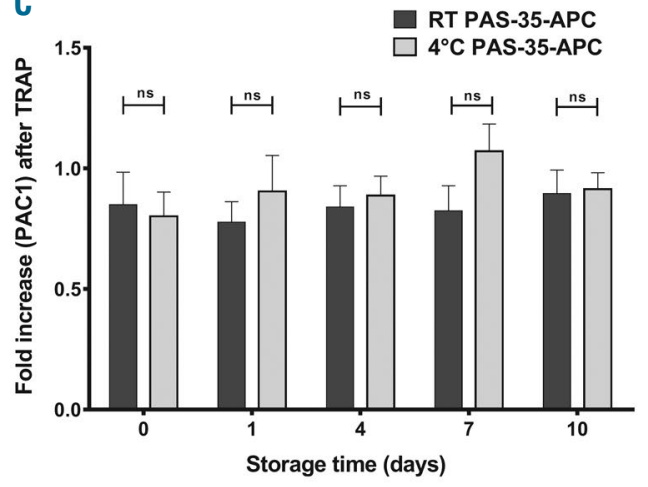

B

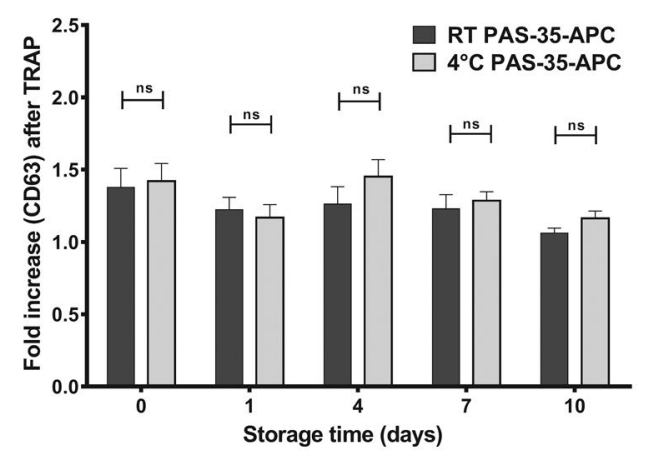

D

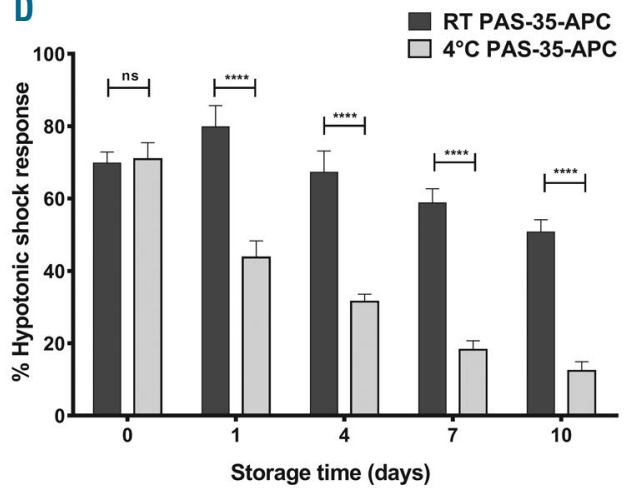

Figure 6. Flow cytometric analysis of apheresis platelet concentrates produced under Good Manufacturing Practice (GMP) conditions. Apheresis platelet concentrates (APCs) were produced under GMP conditions. Platelet (PLT) activation was measured at the indicated storage time for room temperature (RT) and $4^{\circ} \mathrm{C}$ stored PLTs. The expression of (A) CD62P, (B) CD63 and (C) PAC1 was analyzed after activation with TRAP. (D) The ability of apheresis platelet concentrates (APCs) to react on a hypotonic environment was determined as hypotonic shock reaction by light transmission aggregometry. Data are shown as mean \pm Standard Error of Mean. $* * * P<0.001 ; * * * * P<0.0001$. ns: not significant $(\mathrm{n}=4)$.

the von Willebrand factor binding to GPIb- $\alpha$, inducing increased intracellular calcium concentration and phosphatidylserine exposure leading to a rapid clearance of PLTs. The authors demonstrated that the inhibition of this binding and the consequent downstream cascade, using a specific peptide that recognizes GPIb- $\alpha$, significantly increased recovery and life-span of cold-stored PLTs. ${ }^{28}$ Another study showed that a specific caspase-3 inhibitor significantly improves PLT functionality and viability during seven days of storage. ${ }^{29}$ This, together with our results, suggests that inhibition of apoptosis seems to be a promising approach to reduce cold-stored lesions.

Another important finding of our study is that in vivo survival of cold-stored PLTs was independent of varying plasma contents. However, remarkably poor in vitro results of PLT function were observed in APCs stored at too low a plasma concentration (PAS-20-APC). This further supports in vivo studies of Slichter et al. who used radiolabeled autologous PLTs and showed that RT storage in $80 \%$ Plasmalyte (a PAS which is FDA approved) is associated with lower recovery and survival compared to PLTs stored in $100 \%$ plasma or $35 \%$ residual plasma. ${ }^{30}$

Finally, we investigated whether our research findings could be transferred to routine production and explored the possibility of extending PC shelf-life. To exclude the influence of different donors, we designed a follow-up study where each donor donated double APCs in PAS con- taining $35 \%$ plasma. Each APC was stored either at RT or at $4^{\circ} \mathrm{C}$ for up to ten days. We found that PLTs from APCs collected and stored under routine blood bank conditions at $4^{\circ} \mathrm{C}$ maintained functionality better in terms of activation (granule release in response to TRAP) and aggregation compared to those stored at RT. These results correspond to those from the split design study. Taken together, a residual plasma content of approximately $35 \%$ is feasible for cold storage of PLTs in APCs for clinical use. Our results indicate the potential of prolonging shelf-life of GMP-produced APCs stored at $4^{\circ} \mathrm{C}$ for clinical use.

In summary, our study provides additional information on the in vitro hemostatic function and in vivo survival of cold-stored PLTs, and suggests that PLTs stored in PAS at $4^{\circ} \mathrm{C}$ could become an alternative to the current standard of care.

\section{Funding}

The study was supported by a grant from the German Red Cross, Blutspendedienst Baden-Württemberg-Hessen. The authors would like to thank Ulrike Strobel, Lars Jansen, Robert Koch, Flavianna Rigoni, Kati Sevke-Masur and Inga Miksa for their excellent technical support.

\section{Acknowledgments}

We thank Stephen Bosher for his contribution to the manuscript as a native English speaker. 


\section{References}

1 Rosenfeld BA, Herfel B, Faraday N, Fuller A, Braine $\mathrm{H}$. Effects of storage time on quantitative and qualitative platelet function after transfusion. Anesthesiology. 1995;83(6):1167-1172.

2 Brecher ME, Blajchman MA, Yomtovian R, Ness P, AuBuchon JP. Addressing the risk of bacterial contamination of platelets within the United States: a history to help illuminate the future. Transfusion. 2013; 53(1):221-231

3 Dumont LJ, Gulliksson H, van der Meer PF, et al. Interruption of agitation of platelet concentrates: a multicenter in vitro study by the BEST Collaborative on the effects of shipping platelets. Transfusion. 2007;47(9): 1666-1673.

4 Seghatchian J, Krailadsiri P. The platelet storage lesion. Transfus Med Rev. 1997;11 (2):130-144

5 Capocelli KE, Dumont LJ. Novel platelet storage conditions: additive solutions, gas, and cold. Curr Opin Hematol. 2014;21(6): 491-496.

6 Devine DV, Serrano K. The platelet storage lesion. Clin Lab Med. 2010;30(2):475-487.

7 Currie LM, Harper JR, Allan H, Connor J. Inhibition of cytokine accumulation and bacterial growth during storage of platelet concentrates at 4 degrees $C$ with retention of in vitro functional activity. Transfusion. 1997;37(1):18-24.

8 Sandgren P, Hansson M, Gulliksson H, Shanwell A. Storage of buffy-coat-derived platelets in additive solutions at 4 degrees $\mathrm{C}$ and 22 degrees $\mathrm{C}$ : flow cytometry analysis of platelet glycoprotein expression. Vox Sang. 2007;93(1):27-36.

9 Reddoch KM, Pidcoke HF, Montgomery RK, et al. Hemostatic function of apheresis platelets stored at 4 degrees $\mathrm{C}$ and 22 degrees C. Shock. 2014;41 Suppl 1:54-61.

10 Pidcoke HF, Spinella PC, Ramasubramanian $\mathrm{AK}$, et al. Refrigerated platelets for the treatment of acute bleeding: a review of the literature and reexamination of current standards. Shock. 2014;41 Suppl 1:51-53.
11 Montgomery RK, Reddoch KM, Evani SJ, Cap AP, Ramasubramanian AK. Enhanced shear-induced platelet aggregation due to low-temperature storage. Transfusion. 2013;53(7):1520-1530

12 Lee DH, Blajchman MA. Novel treatment modalities: new platelet preparations and substitutes. $\mathrm{Br}$ J Haematol. 2001; 114(3):496-505.

13 Hoffmeister KM, Felbinger TW, Falet $\mathrm{H}$, et al. The clearance mechanism of chilled blood platelets. Cell. 2003;112(1):87-97.

14 Murphy S, Gardner FH. Effect of storage temperature on maintenance of platelet viability--deleterious effect of refrigerated storage. N Engl J Med. 1969;280(20):1094-1098.

15 Pidcoke HF, McFaul SJ, Ramasubramanian AK, et al. Primary hemostatic capacity of whole blood: a comprehensive analysis of pathogen reduction and refrigeration effects over time. Transfusion. 2013;53 Suppl 1:137S-149S

16 Jobes D, Wolfe Y, O'Neill D, et al. Toward a definition of "fresh" whole blood: an in vitro characterization of coagulation properties in refrigerated whole blood for transfusion. Transfusion. 2011;51(1):43-51.

17 Valeri CR. Circulation and hemostatic effectiveness of platelets stored at $4 \mathrm{C}$ or 22 C: studies in aspirin-treated normal volunteers. Transfusion. 1976;16(1):20-23.

18 Sweeney J, Kouttab N, Holme S, Kurtis J, Cheves T, Nelson E. Storage of platelet-rich plasma-derived platelet concentrate pools in plasma and additive solution. Transfusion. 2006;46(5):835-840.

19 Slichter SJ, Corson J, Jones MK, et al. Exploratory studies of extended storage of apheresis platelets in a platelet additive solution (PAS). Blood. 2014;123(2):271-280.

20 Hornsey VS, McColl K, Drummond O, et al. Extended storage of platelets in SSP platelet additive solution. Vox Sang. 2006; 91(1):41-46.

21 Thiele T, Pohler P, Kohlmann T, et al. Tolerance of platelet concentrates treated with UVC-light only for pathogen reduction--a phase I clinical trial. Vox Sang. 2015; 109(1):44-51.

22 Bakchoul T, Fuhrmann J, Chong BH,
Bougie D, Aster R, Subcommittee on Platelet I. Recommendations for the use of the non-obese diabetic/severe combined immunodeficiency mouse model in autoimmune and drug-induced thrombocytopenia: communication from the SSC of the ISTH. J Thromb Haemost. 2015; 13(5):872-875.

23 Fuhrmann J, Jouni R, Alex J, et al Assessment of human platelet survival in the NOD/SCID mouse model: technical considerations. Transfusion. 2016; 56(6):1370-1376.

24 Jansen AJ, Josefsson EC, Rumjantseva V, et al. Desialylation accelerates platelet clearance after refrigeration and initiates GPIbalpha metalloproteinase-mediated cleavage in mice. Blood. 2012;119(5):12631273.

25 Wandall HH, Hoffmeister KM, Sorensen $\mathrm{AL}$, et al. Galactosylation does not prevent the rapid clearance of long-term, 4 degrees C-stored platelets. Blood. 2008;111(6): 3249-3256

26 van der Wal DE, Du VX, Lo KS, Rasmussen JT, Verhoef S, Akkerman JW Platelet apoptosis by cold-induced glycoprotein Ibalpha clustering. I Thromb Haemost. 2010, 8(11):2554-2562.

27 van der Wal DE, Verhoef S, Schutgens RE Peters M, Wu Y, Akkerman JW Role of glycoprotein Ibalpha mobility in platelet function. Thromb Haemost. 2010;103(5):10331043.

28 Chen W, Druzak SA, Wang Y, et al. Refrigeration-Induced Binding of von Willebrand Factor Facilitates Fast Clearance of Refrigerated Platelets. Arterioscle Thromb Vasc Biol. 2017;37(12):2271-2279.

29 Shiri R, Yari F, Ahmadinejad M, Vaeli S, Tabatabaei MR The caspase-3 inhibitor (peptide Z-DEVD-FMK) affects the survival and function of platelets in platelet concentrate during storage. Blood Res. 2014; 49(1):49-53.

30 Slichter SJ, Bolgiano D, Corson J, et al. Extended storage of buffy coat platelet concentrates in plasma or a platelet additive solution. Transfusion. 2014;54(9):22832291. 